\title{
MOTIVACIONES EPISTEMOLÓGICAS SOBRE LAS QUE SE BASAN LAS ELECCIONES DIDACTICAS LLEVADAS A CABO EN LAS ACTIVIDADES EDUCATIVAS EN ITALIA, DESDE PRE-ESCOLAR HASTA EL BIENIO SUPERIOR
}

\author{
D'AMORE, B. \\ Universidad de Bologna, Italia. \\ Traducción castellana de $\mathbf{M}^{\mathrm{a}}$ Jesús Salvador Jiménez
}

\section{SUMMARY}

This article offers a general view of the recent curriculat changes of Mathematics in Italy and discusses the epistemological conceptions underlying the different didactical choices now available or appearing along with curricular changes.

Los años ' 80 se caracterizan, en Italia, por un gran fermento innovador que atañe a todos los niveles escolares. Se trata de una gran tarea: tenovar los programas escolares, anticuados desde hace décadas, acercándolos a los más avanzados de otros países europeos. Se trata de adaptar el programa a una nueva realidad social, tecnológica y científica; esta última, sobre todo, supone el empuje más vigoroso, ya que en el pasado Italia sufrío años de predominio absoluto de la cultura humanística sobre la cientffica, a causa de una estúpida diferenciación entre lo que se dio en llamar "las dos culturas" (Enriques 1938).

Ahora, si es cierto que hay que reconocer peculiaridades e intereses diferentes en tres tipos de cultura (continuaremos pues conservando las denominaciones ya usadas de: "humanística", "tecnológica", y "científica"), también es cierto que no existe una fractura entre las tres, al contrario, precisamente su influencia recíproca y su presencia al mismo tiempo en todos los sectores culturales (en primer lugar: la educación) nos proporcionan un individuo completo, cuya formación personal va de acuerdo con los tiempos.

Veamos ahora un breve panorama de la situación, tal como se presenta en la actualidad.
1) La "scuola materna" (pre-escolar) cada vez está más difundida en Italia, de forma masiva en el Norte, más bien relevante en el Centro y bastante insuficiente en el Sur. Cada vez mayor es la presencia significativa de los docentes de este nivel en los cursos de carácter cultural, y cada vez se nota más la exigencia de profundizar en la competencia, incluso matemática, de estos docentes, ya que los empujes para iniciar un verdadero currículo matemático son notables. Desde hace años, trabaja una comisjón cuya tarea es la de difundir un texto de "Orientación" para esta Escuela no obligatoria (a la que acuden niños de 3 a 5 años) (Caldelli i D'Amore 1986a).

Actualmente, parece que, en ese texto de Ley, también se hablará incluso de "materias" singularizadas, entre ellas las matemáticas; un poco para incitar a que se trabaje en las áreas disciplinares, un poco para frenar las experiencias "saIvajes" que, incontrolables, a veces toman pie y consenso en la base, sin que desde arriba se consiga dar explicaciones plausibles de ello.

Naturalmente no se habla de unas verdaderas "matemáticas" (lo que sería francamente inoportuno), sino que se está difundiendo un nuevo término bastante significativo: "protomatemáticas" (que no hay que confundir 
con pre-matemáticas, término ligado en un pasado reciente a la demagógica y dañina intención de querer a toda costa anticipar nociones matemáticas, en el sentido estricto, anteponiéndolas antes de tiempo y creando, por consiguiente, dificultades al pasar al nivel sucesivo).

2) La Escuela Elemental en Italia es obligatoria para todos; como norma general acuden a ella niños de 6 a 10 años (tiene pues una duración de 5 años). En septiembre de 1987 entraron en vigor nuevos programas ministeriaies que sustituyeron a los precedentes, que llevaban más de 35 años, y que eran sobre todo viejos en la intención. Los nuevos, sin embargo, tienen contenidos que, si bien no son prescriptivos para el docente, ni siquiera como lista de contentidos "mínimos", presentan un grave problema: son "demasiado" nuevos. En ellos se encuentran referencias explícitas a las transformaciones geométricas de todo tipo, a la informática, a la probabilidad, a la lógica... Y esto contrasta con la realidad cultural de base. Para ser docente de la Enseñanza Básica, en Italia, hay que estudiar en Insti tutos Superiores hasta la edad de 17 años y los programas de matemáticas de dichos institutos NO prevén, ni han previsto nunca, contenidos que de alguna manera hagan referencia a las nuevas exigencias. Se están intentando dos vías: A) cursos de formación "fuerte" para maestros con cierta disponibilidad (una media de ocho por provincia) y, al mismo tiempo, cursos más "débiles" para todos; B) experimentos con licenciaturas especiales para maestros de la Escuela Elemental (D’Amore 1986). Es difícil hacer entender que, más allá de la renovación cultural (nuevos contenidos), las matemáticas se proponen hoy de manera completamente nueva (metodológicamente); pero esta instancia de novedad sólo puede recibirla quien se da cuenta de los nuevos contenidos culturales. Se trata de una serpiente que se muerde la cola...

3) En Italia, la enseñanza obligatoria continúa, además de la Escuela Elemental, con la Escuela Media en la que los niños tienen edades comprendidas entre los 11 y los 13-14 años. La Escuela Media, pensada como "conexión" entre los estudios básicos y los estudios avanzados, en diversas ocasiones ha sido objeto de análisis y de varias reflexiones evidentes. Tanto es así que se trata del período escolar que ha sido objeto de la experimentación de nuevos programas en más ocasio* nes. El momento más relevante fue el de la eliminación del latín (1962) y la elección de la Escuela Media como continuación de la enseñanza obligatoria. También fue ocasión para intentar valorar las ciencias; ahora bien, las matemáticas fueron englobadas con todas las cien" cias "naturales", elección bastante discutible, pero todavía vigente hoy en día. Los últimos programas, ya viejos, son de 1979. En ellos se reconoce en las "ana" logías de estructura" la función de eje principal de las matemáticas, se autoriza la presencia oficial del plano cartesiano, transformaciones geométricas, lógica y probabilidad, mientras que faltan referencias explícitas a la informática.

Antes de proseguir con esta exploración vertical, hay que decir que la obligatoriedad escolar en Italia coinci* de con la obtención de la "Licenza di Scuola Media"y, si ésta no se obtiene, se acaba al cumplir los 15 años. Para no obligar a los jóvenes con dificultades escolares a permanecer inútilmente en la Escuela Media más allá de los 15 años, la tendencia más difundida es Ia đe otorgar de todas formas esa "Licenza" para encauzar a los jóvenes con menor disposición al estudio hacia el mundo del trabajo. La fácil obtención de la "Licenza", por el contrario, da falsas ilusiones a los jóvenes y a sus padres sobre las capacidades efectivas, con las obvias consecuencias.

Desde los años ' 60 es cada vez mayor el número de jóvenes que, una vez obtenido el diploma elemental, siguen los estudios, "probando suerte" en el mundo de la Enseñanza Superior, llenando las aulas los primeros años, pero con gran incertidumbre sobre el deseo real de continuar. Así pues, se hace completamente necesaria la organización curricular del bienio superior, como fase escolar en sí misma, antes de ver todo el conjunto de la Enseñanza Superior. En Italia hay muchos tipos de Enseñanza Superior, cuya duración oscila entre los 4 y 5 años; se piensa, en un futuro, uniformarlo a 5 años, distinguiendo, sin embargo, un bienio que quizás sea único para todos, de un trienio que será de dura especialidad profesional. En este momento, el estudio del posible currículo del bienio se encuentra en una fase avan. zada; en particular, la Comisión de matemáticas no sólo ha concluido desde hace tiempo su trabajo, sino que su propuesta ya ha sido avalada.

4) EI bienio superior (que tal vez sea obligatorio para todos) agrupa a estudiantes desde los 14 a los 15-16 años. Las matemáticas han sido divididas en "fuerte" mente presentes" y "débilmente presentes" según a lo que aspire el estudiante. Obviamente es fuerte la presencia de la geometría, hacia cuya revaloración didáctica somos muchos los que estamos empujando en Italia; de la misma manera que definitivamente ha entrado ya oficialmente la lógica, que al inicio había tenido ad. versarios, pero después ha sido debidamente incluida en el primer tema, junto a la informática.

5) Mientras tanto una Comisión está trabajando para el estudio de nuevos programas de matemáticas en el sucesivo trienio superior, según el campo profesional elegido por el estudiante.

6) También hay otra Comisión que trabaja para la reno* vación de la licenciatura en matemáticas; somos ya mu* chos los que consideramos que la dirección "didácti" ca", eso que proveerá al país de futuros docentes de matemáticas, debe ser algo altamente especializado. Por lo que se configura la idea de que el curso de licenciatura en matemáticas para los futuros profesores deberá tener una fisonomía propia que lo caracterice; y que, aun habiendo obtenido una licenciatura en matemáticas, para poder acceder a la enseñanza se tendrán que realizar, en el plan didáctico de los cursos, algunos exámenes que se reconocerán como característica del plan didáctico; parece también (y éstas son las últimas novedades) que para obtener la "capacitación" para en" señar matemáticas, será necesario realizar, tras la li. cenciatura (4 años), otros dos de especialidad en "di- 
dáctica de las matemáticas" con los exámenes correspondiente?

En momentos de gran entusiasmo, como éste que estamos atravesando en Italia, se habla (a veces con desatino) de objetivos didácticos, de experimentos, de contenidos interdisciplinares, de nuevas metodologías, $y$, especialmente desde hace poco tiempo, de los nuevos y sofísticados medios que la tecnología actual nos ofrece.

Sin embargo, a mi modo de ver, se habla demasiado poco de las motivaciones epistemológicas, si existen, que caracterizan a las actuales tendencias: las elecciones didácticas que surgen hoy en día ison fruto de experimentos?, ¿son sólo dictadas por los psicólogos "experimentalistas"?, ¿ son causales? ${ }^{¿}$ son el resultado de la experiencia de los maestros más "avanzados" o sensibles? ¿No se pueden encontrar motivaciones filosoficas, posiblemente escondidas, tras las tendencias que surgen? Si es así, ¿cuáles? Su evidenciación no sería banal; tanto más cuanto en el bienio de especialidad post-licenciatura para futuros docentes de matemáticas, o en los cursos de licenciatura para maestros, en un futuro se podría prever la puesta en marcha de algún curso universitario de carácter filosófico, y es entonces un deber prever estilo y contenidos para que éste no se convierta en el reino de la confusión y de la banalidad... He aquí por qué me parece un deber dar un vistazo a ciertas actitudes que están presentes actualmente (no sólo en Italia) en este campo; al hacerlo, me he encontrado frente a dos posibilidades: 1) seguir un orden "didáctico-curricular", es decir, empezar por la Escuela Materna (Pre-escolar), dando evidencia a las elecciones hechas allí, y proseguir paso a paso; 2) analizar las líneas de pensamiento actualmente presentes entre los "expertos en didáctica de las matemáticas" o, más simplemente, entre los profesores de matemáticas. Si bien la segunda forma parece más confusa, en realidad es la que mejor me permite situarme perfectamente en el tema (D'Amore 1981).

Un actitud bastante difundida, para Ia que es difícil encontrar una "etiqueta" filosófica, es Ia que llamaré "purista". Resumiendo, el "purista" afirma que las matemáticas son lo que son; no se puede "divulgar" el contenido, no se puede esperar "extraer" de las matemáticas una "subteoría" que pueda ir bien a niveles escolares "inferiores": o se "transmiten" tal como son, o es mejor esperar. Si bien este modo de pensar es de los más difundidos, por suerte quien piensa así no tiene ninguna influencia en el campo decisorio. El "purista" no conoce todo el debate que tiene su cumbre en Piaget, o bien solamente ha oído hablar de él, cree que Piaget ha "defendido la teoría de conjuntos"... Incluso en un momento de fuertes críticas a la obra de Piaget, como Ia actual (crítica constructiva, digo), considero que es imposible trabajar en este campo e ignorar la obra que ha condicionado enormenente, y que todavía condicionará por mucho tiempo, nuestras elecciones. Se puede replicar que existe solo una Matemática, que es en efecto un corpus único, macizo y (desde el exterior, para quienes no son especialistas) perfecto. Pero no son éstas las matemáticas que interesan al didacta, éste debe necesariamente tener en cuenta la edad del alumno; de ahí nace, por consiguiente, una didáctica en espiral o "de regresos": el mismo tema no esta del todo concluido en didáctica, sino que, en los diversos niveles críticos en que es posible, regresa; pero regresa a un nivel de profundidad cada vez mayor. Si este "axioma fundamental de la didáctica" no es aceptado, entonces considero oportuno evitar todo tipo de didáctica hasta la Universidad.

Un ejemplo de didáctica de regresos, admitiendo el axioma por el cual es lícito adaptar con oportunas estrategias didácticas las matemáticas a la edad de quien las aprende, es el siguiente, sacado de la geometría.

En primer lugar, en la Escuela Materna (Pre-escolar) y la Escuela Elemental (a veces incluso al principio de la Escuela Media) (Caldelli i D'Amore 1986b), la geometría se reduce esencialmente a una parte de la física. Se trata, grosso modo, de la creación de un lenguaje ade. cuado para describir cuanto sucede en concreto en el mundo empírico. Dos comportamientos profundamente conectados: heurístico y verbal. Se observa, se "ensaya", se hacen hipótesis, se describe, se intenta una regla, se verifica, se falsa, se describe una vez más, y así sucesivamente. Sí, entonces las matemáticas están bastante cerca de una de las tantas ciencias naturales y la geometría, en particular, permite la elección del oportuno vocabulario, cada vez más enriquecedor, para describir el mundo empírico. Se trata de adecuar las experiencias concretas a las elecciones lexicales: ad. quirir un lenguaje que es, de manera especial, geométrico. La descripción es espontánea, aunque obviamente está dirigida; no existe el preliminar de la "definición", seguido a continuación por el ejemplo o por el ejercicio. Aquí, entiendo por definición una lista personal de propiedades características descubiertas por el niño, sin peculiaridades abstractas o formales. Si en tercero ( 8 años) un niño afirma que un cuadrado es un cuadrilátero que tiene los cuatro ángulos iguales, los cuatro lados iguales, las diagonales iguales, que se cortan en la mitad, que son perpendiculares, que tiene cuatro ejes de simetría, y alguna cosa más; yo sé muy bien que ésta es una "definición" de cuadrado demasiado amplia y que, en realidad, una de esas definiciones podría caracterizar por sí sola la figura; pero, de todas formas, me parece muy bien. Precisamente evito una actitud "purista" y acepto la elección espontánea del niño de cubrir una lista (superabundante) de propiedades. Se dará un gran paso hacia adelante cuando, un día, madurado críticamente y por sí solo o empujado de forma oportuna, descubra que algunas de las propiedades caracterizan bien al cuadrado, y que las otras propiedades son una "consecuencia" (palabra mágica, pero difícil, en matemáticas). Se tratará de "enriquecer" el vocabulario geométrico propio, "limpiar" las descripciones propias, pero sintiendo también el gusto hacia estas dos operaciones, no simplemente porque "se hace así" o porque el profesor quiere que se haga así. La naturaleza de la geometría es de carácter crítico: así como las convicciones elementales hacia el modo de ser del mundo son vistas poco a poco por el joven sobre la base de nuevas experiencias y, sobre todo, de nuevas adquisiciones en el campo crítico; del mismo 
modo, en geometría, el joven, un poco mayor, un poco más maduro, un poco más responsable, irá recapacitando sobre sus propias convicciones, que él mismo se verá obligado a revisar para no caer en una contradicción. La elección prioritaria de la evidencia ("se ve que es así"), deberá dejar paso a la derivación lógica. Essto tenderá a suceder en la Escuela Media. Los chicos llegan a ésta todavía muy ligados a la claridad intuitiva de la simple evidencia; pero se desvinculan, si son guiados adecuadamente, en busca de métodos, de lenguajes, de pruebas. Entendámonos bien: en la Escuela Media jtodavía me parece bien que la demostración del enunciado del Teorema de Pitágoras consista en pesar cartulinas, recortadas a ese efecto! Con la condición de que esto esté llevado, por lo menos, como experiencia de laboratorio, con las mismas cautelas científicas de que gozan las otras ciencias (naturales), y no se pretenda que sea una verdadera demostración. Es más, se debería generar en el joven-experimentador la duda sobre la exactitud de tal "comprobación".

En Italia se tiene por costumbre desarrollar un tratamiento hilbertiano de la geometría ya en el primer año de la escuela superior; y es así desde siempre, desde que el tex to base de la geometría ha sido considerado el de Euclides, oportunamente puesto al día. Son muchas las presiones "puristas" que llevan a ello. Pero las más modernas posiciones al respecto pretenden posponer este tratamiento hasta el momento en que el chico sea suficientemente crítico y dueño de la materia, que sea capaz de comprender ese espíritu que no dudaré en llamar hipotético deductivo, ya que esta afortunada denominación tiene su origen en la intuición de un italiano. La "construcción de carácter axiomático" de la geometría, pues, no sólo no aparece como necesaria para su estudio elemental, sino que parece necesario aplazarla hasta otras fases de madurez conceptual. En resumen, es el punto de llegada y en absoluto el punto de partida, como lo fue en la historia. ¡Y que los puristas se condenen por esta elección emergente y sensata!

En Italia, el debate sobre Ia l6gica ha sido violentísimo. En la primera contraposición: "Lógica sí, lógica no", la respuesta ha sido claramente positiva. Hoy en dia se habla de lógica en todos los niveles escolares, desde el pre-escolar hasta la enseñanza superior. Es más, a veces nosotros mismos, que siempre defendimos la presencia explícita de la lógica en los programas, de vez en cuando nos vemos obligados a frenar los fáciles entusiasmos de los neófitos... Pero la nueva interrogación es ahora “¿cómo?". En Pre-escolar, obviamente, y en buena parte de Ia Escuela Elemental, la lógica no es más que una vasta gama de observaciones dirigidas hacia el uso y la función de la lengua natural; de la simple observación, poco a poco nace la lógica como instrumento. Al principio es un instrumento para el análisis, se convierte luego en instrumento de confrontación, al final en instrumento de lengua. Desde la Escuela Elemental, pero ya sin los excesivos énfasis de los conjuntos de otros tiempos, la lógica muestra al alumno su doble papel: 1) la lógica es una parte de las matemáticas, ofrece así en sí misma contenidos que son peculiarmente suyos y que pertenecen al vasto dominio de las matemáticas; 2) la lógica es, una vez se la conoce, un instrumento-método-lenguaje de las matemáticas (que NO se debe confundir con un exceso de formalismo perjudicial y estéril, gratuito y malsano, tal como explicaré mejor más adelante).

Pero solo en la Escuela Media es realmente un instrumento de investigación; mientras que en el bienio superior, gracias también a la estrecha unión que se ha querido ver con la informática, la lógica adquiere definitivamente ese doble papel fundamental suyo. Es más, explícitamente, se hace alusión al hecho de que la lógica es metalenguaje de las matemáticas (se habla de demostrar, de deducir, se citan reglas de inferencia...). Se plantea aquí una nueva cuestión, que en un importante Congreso Nacional del U.M.I. (Unión Matemática Italiana) sobre didáctica, se presentó en estos términos, opuestos a simple vista: 1) la lógica es el primer capítulo de las matemáticas, es decir, antes de empezar a tratar las matemáticas, nos debemos sentir en la necesidad de hacer que los niños aprendan lo que quiere decir razonar; 2) la lógica está insertada en las matemáticas y así, conforme se procede en el estudio de las matemáticas y cada vez que se presente la ocasión espontáneamente, se aprovecha para tratar un poco la lógica (con la esperanza de agrupar todas las matemáticas en el metalenguaje proporcionado por la lógica, al final de los estudios, en que poder mostrar una visión orgánica y compacta de las matemáticas). El ilustre relator, y muchos de los presentes, optaron decididamente por el segundo punto de vista; sin embargo, a mí me parece que se debe optar por un tercer punto de vista que recoja Ios precedentes en un único punto de vista: 3) la logica debe ser el primer capítulo de las matemáticas, pero no debe concluirse en el primer capítulo de las matemáticas; es decir, quien a Ios 14 años asiste a la primera clase superior, posee ya ocho años de aprendizaje de las matemáticas y está preparado a un paso que muy probablemente le ha sido ya inducido por el profesor de la Escuela Media. Tiene la siguiente visión contrastada de las matemáticas: se trata de experiencias concretas que tienen, sin embargo, una base "lógica" escondida entre los pliegues abstractos de la racionalidad. Este es el momento: las matemáticas de la Enseñanza Superior consisten, sobre todo, en mirar al interior de estos pliegues; éste es el primer acercamiento, el resto ya vendrá. No me parece pues contradictorio permitir una aproximación a la lógica, para descubrir después, poco a poco, que se procederá con conexiones y pruebas clarísimas y ejemplares, que los alumnos reconocen $y$ agradecen.

Como se ha visto, ya sea por los ejemplos de la geometría como de la lógica, se deduce un total abandono del pensamiento "purista" en đidáctica, que probablemente se originaba en un encendido filo-euclideismo. Proponemos desde varias partes que las matemáticas sean una evolución que acompañe a los jóvenes: desde unas matemáticas empíricas, bastante cercanas al uso cotidiano, como si se tratara de un instrumento concreto y natural (sobre todo de la lengua común), a unas matemáticas abstractas, hechas de ideas y no de cosas.

Hasta ahora he delineado varios componentes posibles de una epistemología matemática, mirándolos en el 
interior de una disidencia entre los "puristas" y los no "puristas". Sin embargo, surge ahora un nuevo problema quizás menos concreto, pero, eso sí, jmás interesante! ¿Cuáles son, entre las diferentes escuelas filosóficas, las que emergen con mayor prepotencia en todo Io delineado hasta aquí? ¿Qué pensamientos filosóficos contribuyen a la plausibilidad de las elecciones didácticas precedentes? A mi modo de ver, la pregunta no s6lo tiene un sentido, sino que es necesaria desde un punto de vista teórico. Si la única motivación teórica de una elección didáctica (incluso si es empíricamente justificable) fuera la siguiente: “jHemos visto que así, con los niños, funciona!”, consideraría escuálido e infundado el proceso didáctico. Creo que los tiempos han madurado para incluir la Didáctica de las Matemáticas entre las ciencias que emergen. Por supuesto: NO son matemáticas, aunque sean practicadas y teorizadas por matemáticos y no (o mejor, no solamente) por pedagogos. Pero, si es una ciencia, debe tener entonces un sta* tus epistemológico particular, irrenunciable. A eso es legítimo aspirar, como principio general de fundación. A la espera de algo mejor, afirmaré que la didáctica de las matemáticas es una ciencia de carácter empírico que tiene como objeto de estudio el análisis de los procesos cognitivos en relación con las matemáticas. Las elecciones de base de su epistemología, que han sido delineadas anteriormente, se fundan en las diferentes escuelas de filosofía de las matemáticas, según las siguientes observaciones. (En realidad, me parece que un status fundacional real está todavía por llegar y que éstos son sólo los primeros intentos para đistinguir la didáctica de una disciplina de la disciplina, fundando sin embargo la primera sobre las filosofías de base de la segunda).

Entre las actitudes presentes en las primeras fases, como sistemas de base que hay que superar, cualquiera puede reconocer las empiristas. Afirmo explícitamente que parece como si se quisiera admitir que se trata de una elección prioritaria del hombre, en su necesidad de construir un modelo mental y lingǘístico aceptable de todo cuanto le rodea, cosas y acontecimientos.

Inmediatamente se concibe el lenguaje como instrumento idóneo no sólo para enunciar las propias descripciones o las propias observaciones a los demás, sino también para hacerlas inteligibles a nosotros mismos. Por lo que el encuentro pensado/dicho se convierte en una primera fase de superación crítica. No se trata pues de abandonarse a un empirismo excesivo o estrecho, sino de conceder mucho, por lo menos en el plano lingîístico, al logicismo. ¿En qué sentido? Por lo menos en éste: que entre los posibles lenguajes que se pueden elegir para las matemáticas, la elección, poco después, cae en la lógica. Pero, también aquí, no de modo exasperado o totalitario. La lógica elegida como Ienguaje, en primera instancia, no es más que un aspecto, pero el más racional (y concreto) posible, de la lengua natural. De los dos grandes aspectos de la lógica, la logica de los enunciados precede desde todos los puntos de vista a la de los predicados, en la que incluyo los conjuntos. Esa lógica-lenguaje de los conjuntos, poco después, gracias a sus propias peculiaridades de utilidad, asume un papel interesante sobre el que merece la pena hacer consideraciones fundacionales (desde luego, NO desde el punto de vista didáctico, pero reservando la próxima observación a los educadores). Quiero llamar a juicio a la escuela de la teoría de conjuntos. En pocas palabras, ¿qué ha quedado de la "gran revolución" de la teoría de conjuntos de los años 60 ? Sólo las cosas más esenciales y caraterísticas: jla correcta convicción de que un lenguaje que denomine no sólo mediante "nombres individuales" sino también mediante "nombres co. lectivos" vaya en consonancia con el espíritu de las matemáticas! $Y$ que se trata de un lenguaje fácil e indispensable, una vez abandonados (para siempre) mecanismos formales, estúpidamente vacíos e insulsos. Naturalmente, queda por aclarar una cosa que parece natural, ligada a la experiencia, y que constituye sin embargo un axioma metalógico de increíble importancia. Si tengo un universo $U$ y expreso una propiedad individual $P$ (es decir, una propiedad expresada por un predicado monádico), admito que se puede hablar del conjunto $\mathrm{A}$ de los elementos de $\mathrm{U}$ que satisfacen o que hacen verdadera $P$. De ahí en adelante, admitiré que decir "conjunto A" o decir "elementos de U que tienen la propiedad P" es lo mismo. En realidad, debo hacer un esfuerzo mental enorme (a mi modo de ver, uno de los más fuertes de todas las matemáticas) para aceptar lo anterior como un axioma de la teoría de los conjuntos. Esto es normalmente admitido implícitamente en la didáctica de base, pero nunca es bastante considerado por parte de los didactas (quienes, a menudo, creen que es así porque es natural o intuitivo que así sea, ignorando ampliamente los tremendos problemas filos 6 ficos y lógicos que pusieron en "crisis" las matemáticas en un pasado reciente). La naturalidad con que se acepta este axioma fundamental plantea una duda: $\measuredangle$ se trata de la victoria de la posición de la escuela de la teo. ría de los conjuntos ingenua, de la axiomática (que plante 6 realmente axiomas aceptables y "razonables"), o bien solamente de ignorancia?

Varias veces he citado posiciones cuya tínica justificación parece ser la pura intuicion. Pero, a este respecto, seria deshonesto invocar el intuicionismo de Brouwer y de Heyting sólo por asonancia de nombre; es bastante más prudente y correcto pensar en el papel de la intui. ción en el ámbito del đescubrimiento matemático en los dos sentidos de Descartes y Poincaré. En realidad, no obstante, aIgo de intuicionismo en un sentido estrecho alienta en las posiciones didácticas precedentes; y no es tanto el hecho de la determinación de actos simpies de intuición en nuestra mente, en una sucesión tempo. ral que es apriorística; como, en su lugar, jel estrecho constructivismo de las matemáticas que hemos produ* cido! La didáctica se escande según momentos sucesivos de construcción del aprendizaje, ladrillo tras ladri110 ; cada paso es un nuevo ladrillo puesto en su alojamiento a lo largo de una escala de tiempos cronológicamente sucesivos... La lógica que se utiliza en todo esto no es ciertamente de tipo intuicionista brouweriano: se admiten pasos con infinitas sucesiones, demostraciones por reducción al absurdo, recursos a la negación de una negación, etc. Pero queda firme la convicción de que se trata de una verdadera construcción.

Ya he hecho alusión a la presencia de un formalismo 
entre las posibles elecciones fundacionales, incluso en el campo didáctico. Ciertamente, menospreciemos un mito, las matemáticas de alto nivel necesitan el simbolismo y las afirmaciones según las cuales todo cuanto se ha hecho, se está haciendo o se hará en matemáticas no necesita un formalismo, es solo un intento de asombrar al burgués conservador. Sin embargo, en Italia, los nuevos programas por fin hablan claro: Ia identidad presunta y a veces cómoda (para el profesor cansado de buscar soluciones didácticas) "matemáticas = formalismo encendido" está muerta defínitivamente en todos Ios niveles escolares. En el sentido de que no es el exagerado formalismo que acaba en sí mismo, no es el pedante y nefasto recurso continuo al formalismo lo que hace matemáticas; las matemáticas están hechas de ideas, no de signos. Ésta parece ser una buena respuesta a un formalismo encendido, de otros tiempos, que, a Ia larga, ha hecho anteponer toda consideración sintáctica a la semántica, casi como si las matemáticas no se tuvieran que "comprender" sino simplemente aprender por simbiosis, por emulación. Queda obviamente viva la atención ( fundación sintácicica, pero con la condición de que ésta tenga una razón. La razón más correcta y advertida es la siguiente: conviene expresar las matemáticas de modo formal. En resumen: la fórmula es el punto de Ilegada, expresión de una idea adquirida en el fondo, y no es un punto de partida, como sucedía con frecuencia en Italia en el pasado. Un formalismo "fuerte" (primero la sintaxis, luego la semántica y por fin la pragmática) todavía está presente en amplias categorías de profesores en Italia; y ello independientemente de la edad, pero bastante más difundido entre los docentes NO licenciados en matemáticas. Sin extenderme demasiado, ¡está claro que esto se debe al profundo respeto (iterror, miedo...?) que las matemáticas infunden en las mentes de quienes las mastican poco!

También hemos visto que un tema conductor de las matemáticas, tal vez solamente a partir de la Escuela Media en Italia, es el intento de estructurar todo de modo uniforme, reconocer los "tipos de estructuras". Estoy haciendo una llamada explícita a la posición estructuralista, tal vez presente desde siempre, emergente desde las primeras décadas del siglo XIX, triunfante en este siglo con el bourbakismo. En otros países europeos, se inicia desde la más tierna infancia. En Italia es costumbre sugerir el NO hacer emerger explícitamente cuestiones estructurales en la Escuela Elemental, sino sólo a partir de la Escuela Media. Además, es obvio, en caso de que se pueda mostrar de manera clara y simple que existen "conexiones de comportamiento" entre diferentes entidades, que tratan de objetos diferentes, que están unidas por operaciones diferentes, pero que se comportan del mismo modo, entonces jno conviene esperar adrede! Aun habiendo decidido esperar, al menos por parte del profesor habrá un empuje (matemático implícito) para categorizar las diferentes leyes. De otro modo, por ejemplo, al docente no le queda más que la perplejidad sobre la verdadera naturaleza de la geometría como corpus único, aun en la incréble variación de situaciones: signo de que la elección que para nosotros hizo Felix Klein en 1872 se ha revelado como la ganadora (y no sólo en el reino de las geometrías, sino de todas las Matemáticas). Por experiencia sé que los docentes de la Enseñanza Básica aprecian que se les dé a conocer esta cuestión, quizás porque sólo así se consigue dar de nuevo una uniformidad y unitariedad a las facetas de las matemáticas.

Y hablemos ahora del platonismo, en la moderna acepción que tal término está asumiendo.

Abandonado y escarnecido por sus ingenuas posiciones exasperadas, ya sea desde el punto de vista didáctico como epistemológico, resurge nunca dominado, nunca adormecido, nunca definitivamente muerto, un nuevo platonismo. Es más, hay que decir que me parece divisar en este nuevo platonismo la elección filosófica más cercana al corazón de quien hace de la didáctica matemática (y, a menudo, de las propias matemáticas) su propia profesión. Es cierto que el nuevo platonismo ya no tiene la exagerada admisión de una verdadera vida de las ideas, desligadas del mundo empírico que las ha producido. Se contenta con pensar que la verdad no depende de cada una de las verdades que se acumulan juntas, descubrimiento tras descubrimiento; al contrario, cada descubrimiento es una nueva pieza de verdad que se alcanza. No tiene sentido hablar de existencia o, todavía peor, de preexistencia de la verdad, porque se trata de una abstracción pura. Se alcanza cada una de las verdades en el momento en que se descubre el modo de comunicar (a través del lenguaje) ese fragmento de verdad alcanzado. El lenguaje es pues fundamental simplemente para que tenga sentido hablar de verdad. La verdad matemática se expresa, por tanto, mediante un lenguaje oportuno; ese lenguaje es la expresión del propio corpus de las matemáticas y así basa sus propios conceptos en la experiencia. Como si la metamatemática fuera la praxis y las matemáticas su forma lógica, el lenguaje đe la teoría. Es realmente ridículo pensar en una vida pre-existente y en una didáctica hecha de "recuerdos de una vida precedente". Tenía un sentido revolucionario en la época helenística; hoy es perfectamente inútil. Así como tenía sentido el desprecio hacia unas matemáticas hechas de cosas; y esto persiste: no por las cosas en sí mismas, sino como actitud anti-sofista. Las cosas van bien siempre que se admita que aluden a ideas abstractas. Las relaciones entre cosas o entre hechos, o incluso entre palabras, son física o, en todo caso, ciencias naturales; se convierten en matemáticas cuando admiten una desconexión đe la realidad y se hacen abstracción, idea sobre ideas. Ciertamente, como decía poco antes, esta forma de nuevo platonismo debe prestar mucha atención al ienguaje y sobre todo a dos lenguajes: 1) la lengua natural; 2) los lenguajes formales. Mediando el neo-empirismo, en este nuevo platonismo parece que se está dispuesto a admitir la identidad "dicho-pensado" que, nótese, ¡vale en los dos sentidos! Y una mayor disponibilidad por lo que respecta a lo que existe: el mundo será no sólo la totalidad de los hechos, sino también de las cosas que se pueden describir to incluso sólo decir, definir o indicar; y además comprendidas entre las cosas, en este caso, ¿por qué no?, nuevamente las ideas).

Este nuevo platonismo, que parece imperar hoy, sabe distinguir bien entre Sinn y Bedeutung (Frege y Peano); 
sabe plantear distinciones profundas entre verdad de hecho y de razón; entre las ideas que se pueden expresar con palabras convencionales y las asumidas por particulares "cortes de las lenguas maternas", tal como nos han enseñado los antropólogos (Whorf 1956); sabe que existen profundos problemas acerca de la comuni. cación, simplemente científicos o más intrínsecamente psicológicos (Hall 1953, Kristeva 1967). Este nuevo platonismo no se horroriza por los instrumentos, sino que sabe cómo utilizarlos para captar y potenciar con* ceptos, teniendo siempre como referente el mundo de las ideas y de las palabras, a través de las cuales se expresan. Parece que el propio Kurt Gödel, ciertamente uno de los máximos matemáticos de este siglo, había admitido su propio platonismo (Cellucci, ed. 1967); no parece posible que se tratara de un platonismo helenista o asimilable a éste.
En lugar de pensar en un mundo empírico y en unas matemáticas que hacen de conexión entre el empírico y éste, se podrá pensar en una especie de convivencia civil ideal entre el mundo de las cosas y el de las palabras; se podría pensar que existe un isomorfismo estrecho entre el primero y un submundo del segundo...

En el reino de las concepciones didácticas es asi desde hace tiempo; y esto justifica la racionalidad de los pasos, el cuidado que se tiene al hacer progresar, desde un punto de vista abstracto, la mente del nin̄o hacia la del joven, hasta la madurez del conocimiento científico. En caso contrario, Ia frase "a la fuerza", junto a "porque sí", continuarán siendo fórmulas de deducción a las que se recurrirá con frecuencia en el interior de las aulas en las horas de matemáticas.

\section{REFERENCIAS BIBLIOGRÁFICAS}

CALDELLI, M. L. y D'AMORE, B., 1986a. La matematica dalla scuola dell infanzia alla scuola elementare. (La nuova Italia: Firenze).

CALDELLI, M, L. y D'AMORE, B., 1986b. Idee per un laboratorio di matematica nella scuola dell'obligo. (La nuova Italia: Firenze).

CELLUCI, C., (ed.), 1967. La filosofia della matematica. (Laterza: Bari).

D'AMORE, B., 1981. Educazione matematica e sviluppo mentale. (Armando: Roma).

D'AMORE, B., 1986. La didatica della matematica nelia scuola elementare secondo i nuovi programmi. Idee ed esperienze concrete, in D'Amore i Persano, (eds.), Matematica-Scienze. (Armando: Roma).
ENRIQUES, F., 1938. Le matematiche nella storia e nella cultura. (Zanichelli; Bologna).

HALL, E. T., 1953. The silent language. (Publeday: NewYork).

KRISTEVA, I., 1967. Bakhtine, le mot, le dialogue et le roman, Critique, 239. Posteriormente recopilado en J. Kris-

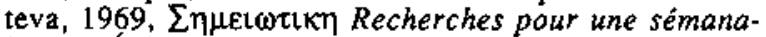
lyse. (Éditions du Seuil: Paris), pp. 143-173. [Trad. castellana, Semiótica. (Akal: Madrid, 1978)].

WHORF, B. L., 1956. Language, Thought and Reality. (MIT Press: Cambridge, Mass) [Trad. castellana, Lenguaje, pensamiento y realidad. (Barral Editores: Barcelona, 1971)]. 